\title{
Modelagem preditiva de distribuição de espécies pioneiras no Estado de Minas Gerais
}

\author{
Guilherme Leite Nunes Coelho(1), Luis Marcelo Tavares de Carvalho(1) e Lucas Rezende Gomide ${ }^{(1)}$
} (1)Universidade Federal de Lavras, Departamento de Ciências Florestais, Caixa Postal 3037, CEP $37200-000$ Lavras, MG, Brasil.
E-mail: guilhermeleite.bio@gmail.com, passarinho@dcf.ufla.br, lucasgomide@dcf.ufla.br

Resumo - O objetivo deste trabalho foi determinar a distribuição potencial de 23 espécies pioneiras no Estado de Minas Gerias, além de identificar as variáveis ambientais que influenciam as suas distribuições. $\mathrm{O}$ algoritmo Maxent foi escolhido para relacionar a ocorrência de espécies às seguintes variáveis bioclimáticas: variação diurna de temperatura, isotermalidade, sazonalidade da temperatura, precipitação do mês mais seco, sazonalidade da precipitação (coeficiente de variação) e evapotranspiração real. Também foram avaliados índice de vegetação por diferença normalizada (NDVI), grau de conservação da flora e heterogeneidade espacial de fitofisionomias, bem como erodibilidade (suscetibilidade do solo à erosão), disponibilidade de água subterrânea, textura do solo, teor de matéria orgânica, ocorrência mineral (espécies minerais existentes por unidade litológica), mapa pedológico simplificado, declividade e altitude. A espécie Anadenanthera colubrina foi a mais indicada para o bioma Caatinga, seguida de Casearia sylvestris e Plathymenia reticulata, indicadas para o bioma Mata Atlântica e Cerrado, respectivamente. Recomenda-se utilizar o Maxent como ferramenta para orientar os planos de conservação que necessitam de indicação de espécies, para recuperar áreas de vegetação degradadas ou desmatadas.

Termos para indexação: Maxent, plano de conservação, planta nativa, predição de habitat.

\section{Predictive modeling distribution of pioneer species in the state of Minas Gerais, Brazil}

\begin{abstract}
The objective of this work was to determine the potential distribution of 23 pioneer species in the state of Minas Gerais, Brazil, as well as to identify the environmental variables that influence their distributions. The Maxent algorithm was chosen to associate the occurrence of species with the following bioclimatic variables: diurnal temperature variation, isothermality, temperature seasonality, driest month precipitation, precipitation seasonality (coefficient of variation), and actual evapotranspiration. The normalized difference vegetation index (NDVI), flora conservation status, and the spatial heterogeneity of vegetation types were also evaluated, besides erodibility (susceptibility of soil to erosion), groundwater availability, soil texture, organic matter content, mineral occurrence (existing mineral species by lithological unit), pedological simplified map, slope and altitude. The species Anadenanthera colubrina was the most suitable for the Caatinga biome, followed by Casearia sylvestris and Plathymenia reticulate, indicated for the Atlantic Forest and the Cerrado biomes, respectively. The use of Maxent is recommended as a tool to guide conservation plans that require the indication of species, aiming to recover degraded or deforested vegetation areas.
\end{abstract}

Index terms: Maxent, conservation plan, native plant, habitat prediction.

\section{Introdução}

A fragmentação de habitats atua como a principal responsável pela perda da biodiversidade em ecossistemas florestais (Gardner et al., 2009). Portanto, diversos esforços de recuperação da vegetação estão sendo realizados para reverter ou limitar os efeitos da fragmentação florestal (Souza et al., 2013). Nos últimos anos, surgiram diversos programas de recuperação florestal para conservação da biodiversidade no âmbito global, nacional e regional (Bouley et al., 2015). Recentemente, também foi lançado o programa "New York Declaration on Forest" que pretende recuperar 150 milhões de hectares mundialmente até 2020 (Bouley et al., 2015).

No Brasil, estudos vêm sendo desenvolvidos com o propósito de criar metodologias específicas de recomposição florestal para cada região do País (Vale et al., 2014). Especificamente em Minas Gerais, tem-se avaliado métodos de recuperação da vegetação nativa 
do estado para subsidiar programas conservacionistas (Souza et al., 2012).

As características das espécies pioneiras, como baixa exigência de solo e crescimento acelerado, são extremamente importantes em estágios iniciais de programas de recuperação florestal, uma vez que tornam o ambiente mais favorável ao desenvolvimento de espécies secundárias e clímax (Gris et al., 2012). No entanto, não há estudos sobre a identificação dos controles ambientais da distribuição espacial de espécies pioneiras usadas em programas de recuperação, nem sobre a estimativa da sua distribuição potencial.

Trabalhos como o de Giacomin et al. (2014), que envolvem a modelagem de distribuição de espécies arbóreas no Brasil, são restritos ao bioma Mata Atlântica. Isso indica que, de modo geral, há uma lacuna de conhecimento sobre as espécies nativas, principalmente em relação à preferência de habitat (Oliveira Filho, 1994), o que limita o uso dessas espécies em programas de revegetação.

Além disso, não há estudos específicos para o estado de Minas Gerais que adotem a modelagem de espécies pioneiras. Trabalhos com essas espécies no estado, como o de Vale et al. (2009), são restritos à autoecologia das arbóreas. Dutra \& Carvalho (2008) conduziram um trabalho de modelagem de distribuição baseada no algoritmo Maxent; entretanto, os autores utilizaram apenas uma espécie indicadora para comparar o desempenho de diferentes algoritmos e diferentes combinações de variáveis independentes.

A modelagem preditiva de distribuição de espécies é um método computacional que constrói uma representação das condições requeridas para a sobrevivência de uma ou mais espécies, por meio da combinação de dados de ocorrência com variáveis ambientais (Anderson et al., 2003). O interesse por esse tipo de ferramenta é crescente, em razão da necessidade de informações rápidas e fundamentadas para direcionar ações conservacionistas, o que evita a perda acelerada da biodiversidade (Giannini, 2012).

O objetivo deste trabalho foi determinar a distribuição potencial de 23 espécies pioneiras no Estado de Minas Gerais, além de identificar as variáveis ambientais que influenciam as suas distribuições.

\section{Material e Métodos}

O estudo foi conduzido no Estado de Minas Gerais, que corresponde a, aproximadamente, $7 \%$ do território brasileiro e ocupa área total de $588.384 \mathrm{~km}^{2}$ na região Sudeste (entre os paralelos $14^{\circ} 13^{\prime} 58^{\prime \prime} \mathrm{S}$ e $22^{\circ} 54^{\prime} 00^{\prime \prime} \mathrm{S}$, e os meridianos $\left.39^{\circ} 51^{\prime} 32^{\prime \prime}, 51^{\circ} 02^{\prime} 35^{\prime \prime} \mathrm{W}\right)$. Minas Gerais abrange oito das nove classes de clima existentes, de acordo com o índice de umidade de Thornthwaite (Carvalho et al., 2008).

Para o estudo, foram selecionadas as 23 espécies pioneiras com maior número de indivíduos e presentes na maioria dos fragmentos. Os registros de ocorrência dessas espécies foram extraídos do Inventário Florestal de Minas Gerais, que consiste no levantamento da flora nativa e de reflorestamento no estado (Mello et al., 2008; Scolforo et al., 2008a, 2008b). Neste inventário, foram levantadas 3.806 parcelas de $1.000 \mathrm{~m}^{2}$ e 650 parcelas de $250 \mathrm{~m}^{2}$, o que representa aproximadamente $0,002 \%$ da área coberta com vegetação nativa em Minas Gerais. Os pontos de ocorrência das espécies utilizadas para a modelagem corresponderam às coordenadas geográficas das parcelas onde as espécies foram observadas.

As variáveis ambientais utilizadas para construir esses tipos de modelo normalmente estão em formato raster ou shapefile, e disponibilizadas em endereços eletrônicos que abrigam grandes bancos de dados produzidos por grupos pesquisadores, como Ambdata (Instituto Nacional de Pesquisas Espaciais, 2013) e Worldclim (Hijmans et al., 2005). Inicialmente, foi realizada uma revisão da literatura para avaliar a qualidade das variáveis disponibilizadas nesses bancos de dados e quais estavam mais relacionadas às preferências de habitat das espécies pioneiras (Peterson \& Nakazawa, 2008).

$\mathrm{Na}$ segunda etapa, foram executados inúmeros modelos-testes com as espécies estudadas. As variáveis com ganhos próximos de zero no teste "jackknife" foram eliminadas. O teste "jackknife" (Pearson et al., 2007) é uma abordagem que exclui uma variável a cada vez que o modelo é executado. Isso permite avaliar qual variável é mais importante para predizer a distribuição da espécie. Uma variável com ganho muito baixo, próximo de zero, apresenta predição tão ruim quanto a de um modelo aleatório. Já variáveis com ganhos próximos a 1 apresentam informações altamente correlacionadas às ocorrências e executam boas predições. O teste "jackknife" foi aplicado para avaliar a importância das variáveis para os modelos de cada espécie, ou seja, qual variável apresenta maior influência na distribuição das espécies (Phillips \& Dudík, 2008). 
Utilizaram-se as informações climáticas disponibilizadas pelo banco de dados Worldclim (Hijmans et al., 2005). As variáveis bioclimáticas variação diurna de temperatura, isotermalidade, sazonalidade da temperatura, precipitação do mês mais seco e sazonalidade da precipitação (coeficiente de variação) foram selecionadas para a modelagem. A evapotranspiração real foi extraída do projeto "Consortium for Spatial Information" (CGIAR-CSI) (Trabucco \& Zomer, 2010).

Também foiavaliadooíndicede vegetaçãopordiferença normalizada (NDVI), extraído do sensor "Moderate Resolution Imaging Spectroradiometer" (Modis), com resolução radiométrica de 16 bits, resolução espacial de 250 m e composição de 16 dias (National Aeronautics and Space Administration, 2014). De acordo com Huete et al. (2002), o NDVI é um bom indicador de biomassa da vegetação, para auxiliar os modelos na identificação da relação entre as espécies e a densidade de vegetação. Foram selecionadas quatro imagens correspondentes às estações do ano, nas seguintes datas: 21/3, 25/6, 29/9 e 18/12, para representar o comportamento espectral sazonal da vegetação. As imagens são referentes a 2004, quando foi realizado o inventário florestal.

Ainda foram analisadas as variáveis topográficas altitude e declividade, que foram extraídas do projeto Ambdata (Instituto Nacional de Pesquisas Espaciais, 2013), o qual utilizou dados da "Shuttle Radar Topographic Mission" (SRTM).

O zoneamento ecológico-econômico, realizado pela Universidade Federal de Lavras, disponibilizou as variáveis grau de conservação da flora, heterogeneidade espacial de fitofisionomias, erodibilidade (suscetibilidade do solo à erosão), ocorrência mineral (espécies minerais existentes por unidade litológica), disponibilidade de água subterrânea, textura do solo, teor de matéria orgânica e mapa pedológico simplificado (Carvalho et al., 2008).

Uma reamostragem dos pixels de todas as variáveis foi realizada para cada variável, tendo-se utilizado resolução espacial de $270 \mathrm{~m}$.

O algoritmo utilizado, implementado na plataforma Java, foi o Maxent, versão 3.3.3k (Princeton University, Princeton, NJ, EUA), que utiliza o método de máxima entropia (Phillips et al., 2006). Os parâmetros iniciais considerados foram os recomendados na literatura: limite de convergência de 0,00001 ; máxima iteração de 500; autorrecursos equivalentes a sim; multiplicador de regularização igual a 1 ; regra aplicada de limite correspondente à mínima presença de treino; número de repetições igual a 10; e limites de adequabilidade baixa (0-1), média (1-66) e alta (66-100) (Pearson et al., 2007). Os mapas analisados para discussão dos resultados foram as médias obtidas pelas repetições.

A ferramenta ENMTools, versão 1.4.3 (Warren et al., 2008), permitiu comparar as estimativas de adequabilidade de habitat calculadas pelo Maxent, para cada quadrante na área de estudo. Isso permitiu quantificar a similaridade das distribuições das espécies, tendo-se avaliado se, em cada quadrante, a adequabilidade de habitat apresentava os mesmos valores para diferentes espécies.

A avaliação do algoritmo Maxent foi realizada por uma técnica chamada de curva "receiver operating characteristics" (ROC). A Scurva é feita ao se plotar, em um eixo, a proporção de presenças verdadeiras do total de presenças preditas e a especificidade e, no outro eixo, a proporção de ausências verdadeiras em relação às ausências preditas. A área abaixo da curva ROC é conhecida como área abaixo da curva (AUC). Valores próximos de 1 indicam que o modelo apresentou alto desempenho; no entanto, valores próximos de 0,5 indicam que o desempenho foi baixo e que a classificação está próxima de uma classificação aleatória (Elith et al., 2006).

\section{Resultados e Discussão}

As curvas ROC dos modelos gerados pelo algoritmo Maxent sempre apresentaram valores acima de 0,9, o que indica boa acurácia (Elith et al., 2006) (Tabela 1). Outros estudos também mostraram que, de fato, o algoritmo Maxent geralmente fornece bons resultados (Elith et al., 2006; Phillips et al., 2006).

Tabela 1. Avaliação do desempenho dos modelos baseados no algoritmo Maxent, com uso da área abaixo da curva (AUC).

\begin{tabular}{lccc}
\hline Espécie & AUC & Espécie & AUC \\
\hline Acosmium dasycarpum & 0,963 & Myrcia splendens & 0,935 \\
Anadenanthera colubrina & 0,941 & Myrsine umbellata & 0,964 \\
Bowdichia virgilioides & 0,945 & Ocotea corymbosa & 0,964 \\
Casearia sylvestris & 0,937 & Plathymenia reticulata & 0,948 \\
Copaifera langsdorffii & 0,965 & Qualea grandiflora & 0,948 \\
Cupania vernalis & 0,966 & Qualea multiflora & 0,954 \\
Dimorphandra mollis & 0,961 & Qualea parviflora & 0,952 \\
Eugenia dysenterica & 0,953 & Roupala montana & 0,940 \\
Eugenia florida & 0,944 & Senegalia polyphylla & 0,945 \\
Hymenaea stigonocarpa & 0,955 & Tapirira guianensis & 0,935 \\
Lafoensia vandelliana & 0,948 & Tapirira obtusa & 0,965 \\
Machaerium brasiliense & 0,955 & & - \\
\hline
\end{tabular}

Pesq. agropec. bras., Brasília, v.51, n.3, p.207-214, mar. 2016 DOI: $10.1590 / \mathrm{S} 0100-204 X 2016000300002$ 
Os pares de espécies cujos nichos apresentaram maior similaridade foram: Qualea grandiflora Mart. e Qualea parviflora Mart. (Figura 1); Q. grandiflora e Eugenia dysenterica DC; e Hymenaea stigonocarpa Mart. ex Hayne e E. dysenterica, com coeficientes de similaridade que atingiram, respectivamente, 0,87, 0,86 e 0,84 . Já os pares de espécies cujos nichos apresentaram menor similaridade foram: $Q$. parviflora e Tapirira obtusa (Benth.) J.D.Mitch.; E. dysenterica e T. obtusa; e E. dysenterica e Ocotea corymbosa (Meisn.) Mez., com coeficientes de similaridade que atingiram, respectivamente, $0,11,0,11$ e 0,12 .

As variáveis que definem os nichos das espécies de acordo com o teste "jackknife" estão descritas na Tabela 2. A variável ocorrência mineral mostrou maior ganho em $74 \%$ dos modelos gerados, com ganho médio de 0,94 , seguida pelo NDVI, com ganho médio de 0,72 , em $26 \%$ dos modelos. O ganho médio das variáveis restantes variou entre 0,41 e 0,56 . Para exemplificar esses resultados, as espécies $O$. corymbosa e Myrsine umbellata Mart. apresentaram valores mais altos para a variável NDVI, que foi diretamente proporcional à adequabilidade das espécies.

A qualidade do solo, representada pela fertilidade, e a densidade da vegetação são fatores importantes para a distribuição das espécies pioneiras. Isso pode ser exemplificado pela maior influência da ocorrência mineral na distribuição das espécies $T$. obtusa e M. umbellata (Tabela 3). Conforme Lorenzi (2002), a primeira espécie, pertencente à família Anacardiaceae, está relacionada a solos de alta drenagem e baixa fertilidade química, enquanto a segunda é uma espécie muito frequente na Mata Atlântica e apresenta pouca exigência de solo e umidade (Lorenzi, 1998).

As três espécies com maiores adequabilidades no bioma Caatinga são Anadenanthera colubrina (Vell.) Brenan, Senegalia polyphylla (DC.) Britton \& Rose in Britton \& Killip e Eugenia florida DC. (Tabela 4). Das 23 espécies estudadas, a primeira espécie apresentou a maior área de alta adequabilidade (591 $\mathrm{km}^{2}$ ) e a quarta maior área de média adequabilidade $\left(8.402 \mathrm{~km}^{2}\right)$. A segunda espécie é considerada uma

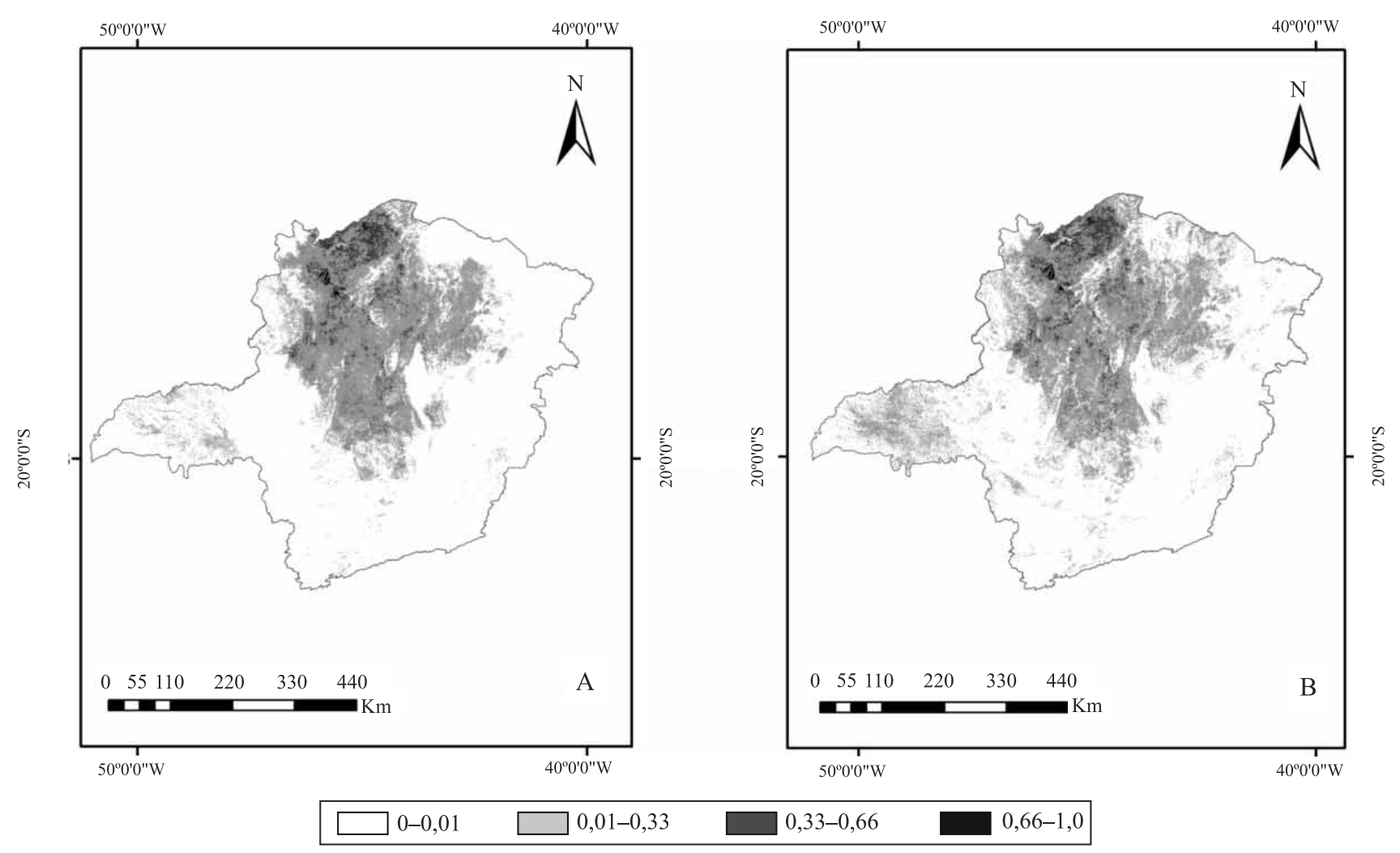

Figura 1. Mapas de probabilidade de ocorrência de Qualea parviflora (A) e Qualea grandiflora (B) no Estado de Minas Gerais, baseados na modelagem preditiva Maxent. 
árvore pioneira de ampla distribuição (Silva et al., 2012) e apresentou área de alta e média adequabilidades, de 500 e $9.100 \mathrm{~km}^{2}$, respectivamente. A terceira espécie apresentou áreas de alta e média adequabilidades, tendo atingido, respectivamente, 407 e $9.219 \mathrm{~km}^{2}$ para o bioma.

No bioma Mata Atlântica, as espécies Casearia sylvestris Swartz (Salicaceae) e Myrcia splendens (Sw.) DC. (Myrtaceae) apresentaram as maiores áreas de alta e média adequabilidades, de $1.791 \mathrm{e}$ $150.065 \mathrm{~km}^{2}$ para a primeira, e de 1.493 e $192.902 \mathrm{~km}^{2}$ para a segunda espécie, respectivamente (Tabela 5). De acordo com Lorenzi (1998), essas duas árvores são indicadas para recuperação de áreas de florestas perturbadas. No presente trabalho, duas das espécies indicadas para recuperação de áreas perturbadas, para o bioma Caatinga, também se destacaram para o bioma Mata Atlântica: E. florida e A. colubrina, que apresentaram áreas de alta adequabilidade superiores à $1.000 \mathrm{~km}^{2}$.
Para o bioma Cerrado, 18 espécies foram associadas a áreas de adequabilidade média, superiores a $150.000 \mathrm{~km}^{2}$, e 13 espécies apresentaram alta adequabilidade em área superior a $1.000 \mathrm{~km}^{2}$. Os valores máximos corresponderam às espécies Plathymenia reticulata Benth. (Fabaceae: Mimosoideae), Lafoensia vandelliana DC. ex Cham. \& Schltdl. (Lythraceae) e Roupala montana Aubl. (Proteaceae), tendo atingido, respectivamente, 2.209, 2.193 e $1.894 \mathrm{~km}^{2}$ (Tabela 6). A primeira espécie ocorre predominantemente em formações abertas de Cerrado e de transição savana-floresta (Lorenzi, 2002); enquanto a segunda é considerada espécie característica de Cerrado e é encontrada em remanescentes do bioma (Pilon et al., 2015).

Os resultados obtidos no presente trabalho permitem nortear e aumentar a chance de sucesso de programas de recuperação de áreas perturbadas, além de contribuir para o aumento do conhecimento científico sobre características ecológicas e preferências de habitat das espécies arbóreas estudadas.

Tabela 2. Valores de ganho para cada variável independente e espécie estudadas, obtidos pelo teste "jackknife" no método de modelagem Maxent.

\begin{tabular}{|c|c|c|c|c|c|c|c|c|}
\hline Espécie & ETra & $\mathrm{Bio} 2$ & Bio14 & Bio15 & Con.fl. & NDVI & Oc.mi. & $\mathrm{pH}$ \\
\hline Acosmium dasycarpum & 0,71 & 0,87 & 0,66 & 0,63 & 0,60 & 0,43 & $\underline{0,95}^{(1)}$ & 0,54 \\
\hline Anadenanthera colubrina & 0,17 & 0,10 & 0,19 & 0,29 & 0,55 & 0,63 & $\underline{0,73}$ & 0,30 \\
\hline Bowdichia virgilioides & 0,58 & 0,57 & 0,46 & 0,48 & 0,64 & 0,55 & $\underline{0,84}$ & 0,45 \\
\hline Casearia sylvestris & 0,23 & 0,06 & 0,11 & 0,20 & 0,41 & $\underline{0,93}$ & 0,91 & 0,56 \\
\hline Copaifera langsdorffii & 0,14 & 0,13 & 0,09 & 0,14 & 0,45 & 0,77 & $\underline{1,01}$ & 0,42 \\
\hline Cupania vernalis & 0,43 & 0,20 & 0,36 & 0,42 & 0,48 & 1,07 & $\underline{1,13}$ & 0,84 \\
\hline Dimorphandra mollis & 0,77 & 0,77 & 0,72 & 0,67 & 0,66 & 0,50 & $\underline{1,00}$ & 0,63 \\
\hline Eugenia dysenterica & 0,70 & 0,73 & 0,77 & 0,74 & 0,67 & 0,43 & $\underline{1,02}$ & 0,65 \\
\hline Eugenia florida & 0,12 & 0,05 & 0,12 & 0,20 & 0,49 & 0,86 & $\underline{0,96}$ & 0,40 \\
\hline Hymenaea stigonocarpa & 0,64 & 0,73 & 0,69 & 0,69 & 0,66 & 0,51 & $\underline{1,01}$ & 0,61 \\
\hline Lafoensia vandelliana & 0,53 & 0,59 & 0,61 & 0,57 & 0,60 & 0,45 & $\underline{0,99}$ & 0,60 \\
\hline Machaerium brasiliense & 0,41 & 0,33 & 0,27 & 0,38 & 0,66 & $\underline{1,14}$ & 0,89 & 0,85 \\
\hline Myrcia splendens & 0,17 & 0,09 & 0,17 & 0,13 & 0,50 & $\underline{0,93}$ & 0,68 & 0,41 \\
\hline Myrsine umbellata & 0,37 & 0,25 & 0,44 & 0,38 & 0,51 & $\underline{1,40}$ & 1,20 & 0,96 \\
\hline Ocotea corymbosa & 0,35 & 0,28 & 0,37 & 0,37 & 0,45 & $\underline{1,22}$ & 0,88 & 0,63 \\
\hline Plathymenia reticulata & 0,59 & 0,60 & 0,48 & 0,49 & 0,57 & 0,36 & $\underline{0,89}$ & 0,44 \\
\hline Qualea grandiflora & 0,64 & 0,75 & 0,65 & 0,64 & 0,63 & 0,45 & $\underline{0,95}$ & 0,57 \\
\hline Qualea multiflora & 0,70 & 0,84 & 0,61 & 0,63 & 0,58 & 0,57 & $\underline{0,98}$ & 0,66 \\
\hline Qualea parviflora & 0,75 & 0,83 & 0,70 & 0,74 & 0,61 & 0,40 & $\underline{1,01}$ & 0,69 \\
\hline Roupala montana & 0,28 & 0,24 & 0,16 & 0,17 & 0,56 & 0,68 & $\underline{0,73}$ & 0,24 \\
\hline Senegalia polyphylla & 0,18 & 0,20 & 0,23 & 0,35 & 0,44 & 0,47 & $\underline{1,00}$ & 0,31 \\
\hline Tapirira guianensis & 0,24 & 0,09 & 0,08 & 0,10 & 0,52 & $\underline{0,81}$ & 0,70 & 0,23 \\
\hline Tapirira obtusa & 0,39 & 0,19 & 0,46 & 0,53 & 0,45 & 1,08 & $\underline{1,22}$ & 0,94 \\
\hline
\end{tabular}

${ }^{(1)}$ Os maiores valores de ganho por modelo estão sublinhado. ETra, evapotranspiração real anual; Bio2, variação diurna de temperatura; Bio14, precipitação do mês mais seco; Bio15, sazonalidade da temperatura; Con.fl., grau de conservação da flora; NDVI, índice de vegetação por diferença normalizada; Oc.mi., ocorrência mineral; e pH, pH da água subterrânea. 
Tabela 3. Valores das variáveis que atingiram probabilidade máxima para as 23 espécies estudadas.

\begin{tabular}{|c|c|c|c|}
\hline Espécie & Ocorrência mineral & NDVI & $\mathrm{Bio} 2^{(1)}$ \\
\hline Acosmium dasycarpum & Arenito, pelito, arenito conglomerático & 6.500 & 145 \\
\hline Anadenanthera colubrina & Filito, quartzito, metagrauvaca, mica xisto & 8.100 & 95 \\
\hline Bowdichia virgilioides & Calcário, metapelito & 6.200 & 145 \\
\hline Casearia sylvestris & Trondhjemito, ortognaisse & 8.200 & 136 \\
\hline Copaifera langsdorffii & Quartzito, mica xisto, xisto aluminoso & 6.700 & 132 \\
\hline Cupania vernalis & Paragnaisse, mica xisto, biotita gnaisse & 8.200 & 138 \\
\hline Dimorphandra mollis & Arenito, pelito, arenito conglomerático & 6.100 & 145 \\
\hline Eugenia dysenterica & Arenito, pelito, arenito conglomerático & 6.100 & 145 \\
\hline Eugenia florida & Quartzito, quartzito ferruginoso & 8.100 & 95 \\
\hline Hymenaea stigonocarpa & Arenito, pelito, arenito conglomerático & 6.100 & 145 \\
\hline Lafoensia vandelliana & Arenito, pelito, arenito conglomerático & 6.100 & 140 \\
\hline Machaerium brasiliense & Trondhjemito, ortognaisse & 8.300 & 105 \\
\hline Myrcia splendens & Granito, trondhjemito & 8.100 & 95 \\
\hline Myrsine umbellata & Granito, trondhjemito & 8.200 & 125 \\
\hline Ocotea corymbosa & Granito, trondhjemito & 8.300 & 95 \\
\hline Plathymenia reticulata & Arenito, pelito, arenito conglomerático & 8.300 & 145 \\
\hline Qualea grandiflora & Arenito, pelito, arenito conglomerático & 6.100 & 145 \\
\hline Qualea multiflora & Aglomerado, areia, argila, laterita & 6.100 & 145 \\
\hline Qualea parviflora & Arenito, pelito, arenito conglomerático & 6.100 & 145 \\
\hline Roupala montana & Calcarenito, calcário, siltito & 8.200 & 145 \\
\hline Senegalia polyphylla & Quartzito, quartzito ferruginoso & 8.600 & 143 \\
\hline Tapirira guianensis & Trondhjemito, ortognaisse & 8.200 & 115 \\
\hline Tapirira obtusa & Granodiorito, tonalito, trondhjemito & 8.200 & 92 \\
\hline
\end{tabular}

${ }^{(1)} \mathrm{Bio} 2$, variação diurna de temperatura.

Tabela 4. Superfície (em milhares de $\mathrm{km}^{2}$ ) coberta pelas classes de adequabilidade por espécie e para o bioma Caatinga, a partir das probabilidades estimadas pelo modelo Maxent para 23 espécies pioneiras.

\begin{tabular}{lccc}
\hline Espécie & \multicolumn{3}{c}{ Superfície para adequabilidade } \\
\cline { 2 - 4 } & Baixa & Média & Alta \\
\hline Acosmium dasycarpum & 1,7 & 9,1 & 0,0 \\
Anadenanthera colubrina & 1,8 & 8,4 & 0,6 \\
Bowdichia virgilioides & 3,8 & 6,9 & 0,0 \\
Casearia sylvestris & 9,1 & 1,7 & 0,0 \\
Copaifera langsdorffi & 7,1 & 3,6 & 0,0 \\
Cupania vernalis & 10,4 & 0,4 & 0,0 \\
Dimorphandra mollis & 8,6 & 2,2 & 0,0 \\
Eugenia dysenterica & 5,1 & 5,7 & 0,0 \\
Eugenia florida & 1,2 & 9,2 & 0,4 \\
Hymenaea stigonocarpa & 6,4 & 4,3 & 0,0 \\
Lafoensia vandelliana & 4,4 & 6,4 & 0,0 \\
Machaerium brasiliense & 7,7 & 3,1 & 0,0 \\
Myrcia splendens & 3,3 & 7,4 & 0,0 \\
Myrsine umbellata & 8,7 & 2,1 & 0,0 \\
Ocotea corymbosa & 10,7 & 0,1 & 0,0 \\
Plathymenia reticulata & 2,1 & 8,6 & 0,0 \\
Qualea grandiflora & 7,3 & 3,5 & 0,0 \\
Qualea multiflora & 9,6 & 1,2 & 0,0 \\
Qualea parviflora & 7,8 & 2,9 & 0,0 \\
Roupala montana & 6,8 & 4,0 & 0,0 \\
Senegalia polyphylla & 1,2 & 9,1 & 0,5 \\
Tapirira guianensis & 6,0 & 4,8 & 0,0 \\
Tapirira obtusa & 10,8 & 0,0 & 0,0 \\
\hline
\end{tabular}

Tabela 5. Superfície (em milhares de $\mathrm{km}^{2}$ ) coberta pelas classes de adequabilidade por espécie e para o bioma Mata Atlântica, a partir das probabilidades estimadas pelo modelo Maxent para 23 espécies pioneiras.

\begin{tabular}{lccc}
\hline Espécie & \multicolumn{3}{c}{ Superfície para adequabilidade } \\
\cline { 2 - 4 } & Baixa & Média & Alta \\
\hline Acosmium dasycarpum & 215,6 & 24,7 & 0,0 \\
Anadenanthera colubrina & 102,1 & 136,9 & 1,3 \\
Bowdichia virgilioides & 170,6 & 69,7 & 0,0 \\
Casearia sylvestris & 88,4 & 150,1 & 1,8 \\
Copaifera langsdorffii & 107,1 & 132,4 & 0,6 \\
Cupania vernalis & 127,3 & 112,6 & 0,3 \\
Dimorphandra mollis & 225,7 & 14,5 & 0,0 \\
Eugenia dysenterica & 225,9 & 14,3 & 0,0 \\
Eugenia florida & 101,7 & 137,1 & 0,1 \\
Hymenaea stigonocarpa & 218,0 & 22,2 & 0,0 \\
Lafoensia vandelliana & 180,7 & 59,6 & 0,0 \\
Machaerium brasiliense & 140,5 & 99,4 & 0,0 \\
Myrcia splendens & 45,8 & 192,9 & 1,5 \\
Myrsine umbellata & 130,1 & 109,9 & 0,0 \\
Ocotea corymbosa & 128,7 & 111,1 & 0,0 \\
Plathymenia reticulata & 160,6 & 79,6 & 0,0 \\
Qualea grandiflora & 223,7 & 16,5 & 0,0 \\
Qualea multiflora & 214,6 & 25,6 & 0,0 \\
Qualea parviflora & 229,8 & 10,5 & 0,0 \\
Roupala montana & 124,4 & 115,7 & 0,0 \\
Senegalia polyphylla & 133,5 & 106,7 & 0,0 \\
Tapirira guianensis & 101,4 & 138,3 & 0,0 \\
Tapirira obtusa & 127,4 & 112,6 & 0,0 \\
\hline
\end{tabular}


Tabela 6. Superfície (em milhares de $\mathrm{km}^{2}$ ) coberta pelas classes de adequabilidade por espécie e para o bioma Cerrado, a partir das probabilidades estimadas pelo modelo Maxent para 23 espécies pioneiras.

\begin{tabular}{lccc}
\hline Espécie & \multicolumn{3}{c}{ Superfície para adequabilidade } \\
\cline { 2 - 4 } & Baixa & Média & Alta \\
\hline Acosmium dasycarpum & 151,6 & 176,7 & 1,0 \\
Anadenanthera colubrina & 172,4 & 155,7 & 1,0 \\
Bowdichia virgilioides & 132,9 & 195,2 & 1,1 \\
Casearia sylvestris & 129,9 & 198,7 & 0,0 \\
Copaifera langsdorffi & 166,1 & 162,7 & 0,0 \\
Cupania vernalis & 195,1 & 134.0 & 0,0 \\
Dimorphandra mollis & 169,9 & 158,2 & 1,1 \\
Eugenia dysenterica & 173,1 & 154,7 & 1,3 \\
Eugenia florida & 163,2 & 165,5 & 0,0 \\
Hymenaea stigonocarpa & 166,6 & 161,4 & 1,1 \\
Lafoensia vandelliana & 139,9 & 187,1 & 2,1 \\
Machaerium brasiliense & 205,5 & 123,6 & 0,0 \\
Myrcia splendens & 87,8 & 239,9 & 1,5 \\
Myrsine umbellata & 220,4 & 108,7 & 0,0 \\
Ocotea corymbosa & 225,0 & 103,9 & 0,0 \\
Plathymenia reticulata & 131,1 & 195,9 & 2,2 \\
Qualea grandiflora & 160,1 & 167,8 & 1,3 \\
Qualea multiflora & 169,4 & 159,2 & 0,0 \\
Qualea parviflora & 168,3 & 159,8 & 1,1 \\
Roupala montana & 117,7 & 209,6 & 1,9 \\
Senegalia polyphylla & 162,8 & 164,8 & 1,5 \\
Tapirira guianensis & 107,1 & 220,8 & 1,2 \\
Tapirira obtusa & 230,0 & 99,1 & 0,0 \\
\hline & & &
\end{tabular}

\section{Conclusões}

1. O índice de similaridade mostra que, entre as espécies estudadas, nenhuma apresenta nicho idêntico a outra, ou seja, cada espécie apresenta padrão de distribuição diferente.

2. As duas variáveis mais influentes na distribuição de espécies pioneiras no Estado de Minas Gerais são a ocorrência mineral e o índice de vegetação por diferença normalizada (NDVI).

3. A modelagem preditiva de distribuição indica espécies mais adequadas para cada bioma do Estado de Minas Gerais, e permite relacionar as características do bioma às preferências ambientais de cada espécie.

\section{Agradecimentos}

À Coordenação de Aperfeiçoamento de Pessoal de Nível Superior (Capes), pela concessão de bolsa.

\section{Referências}

ANDERSON, R.P.; LEW, D.; PETERSON, A.T. Evaluating predictive models of species' distributions: criteria for selecting optimal models. Ecological Modelling, v.162, p.211-232, 2003. DOI: 10.1016/S0304-3800(02)00349-6.

BOULEY, P.; CALLE, A.; CRANDALL, S.G.; DAVENPORT, R.B.; HOLL, K.D.; JENKINS, J.S.; LESAGE, J.C.; OLIMPI, E.M.; OLIVER, C.L.; SKIKNE, S.A.; STANFORD, B. Large-scale forest restoration. Restoration Ecology, v.23, p.501-502, 2015. DOI: $10.1111 /$ rec.12239.

CARVALHO, L.M.T. de; LOUZADA, J.N.C.; SCOLFORO, J.R.; OLIVEIRA, A.D. de. Flora. In: SCOLFORO, J.R.; CARVALHO, L.M.T. de; OLIVEIRA, A.D. de (Ed.). Zoneamento ecológico-econômico do Estado de Minas Gerais: componentes geofísico e biótico. Lavras: UFLA, 2008. p.137-150.

DUTRA, G.C.; CARVALHO, L.M.T. de. Modelos de distribuição geográfica de Amaioua guianensis Aubl. em Minas Gerais, Brasil. Ambiência, v.4, p.47-55, 2008. Edição Especial.

ELITH, J.; GRAHAM, C.H.; ANDERSON, R.P.; DUDÍK, M.; FERRIER, S.; GUISAN, A.; HIJMANS, R.J.; HUETTMANN, F.; LEATHWICK, J.R.; LEHMANN, A.; LI, J.; LOHMANN, L.G.; LOISELLE, B.A.; MANION, G.; MORITZ, C.; NAKAMURA, M.; NAKAZAWA, Y.; OVERTON, J.M.; PETERSON, A.T.; PHILLIPS, S.J.; RICHARDSON, K.; SCACHETTI-PEREIRA, R.; SCHAPIRE, R.E.; SOBERÓN, J.; WILLIAMS, S.; WISZ, M.S.; ZIMMERMANN, N.E. Novel methods improve prediction of species' distributions from occurrence data. Ecography, v.29, p.129-151, 2006. DOI: 10.1111/j.2006.0906-7590.04596.x.

GARDNER, T.A.; BARLOW, J.; CHAZDON, R.; EWERS, R.M.; HARVEY, C.A.; PERES, C.A.; SODHI, N.S. Prospects for tropical forest biodiversity in a human-modified world. Ecology Letters, v.12, p.561-582, 2009. DOI: 10.1111/j.1461-0248.2009.01294.x.

GIACOMIN, L.L.; KAMINO, L.H.Y.; STEHMANN, J.R. Speeding up the discovery of unknown plants: a case study of Solanum (Solanaceae) endemics from the Brazilian Atlantic Forest. Boletim do Museu de Biologia Mello Leitão. Nova Série, v.35, p.121-135, 2014.

GIANNINI, T.C.; SIQUEIRA, M.F.; ACOSTA, A.L.; BARRETO, F.C.C.; SARAIVA,A.M.;ALVES-DOS-SANTOS, I. Desafios atuais da modelagem preditiva de distribuição de espécies. Rodriguésia, v.63, p.733-749, 2012. DOI: 10.1590/S2175-78602012000300017.

GRIS, D.; TEMPONI, L.G.; MARCON, T.R. Native species indicated for degraded area recovery in Western Paraná, Brazil. Revista Árvore, v.36, p.113-125, 2012. DOI: 10.1590/ S0100-67622012000100013.

HIJMANS, R.J.; CAMERON, S.E.; PARRA, J.L.; JONES, P.G.; JARVIS, A. Very high resolution interpolated climate surfaces for global land areas. International Journal of Climatology, v.25, p.1965-1978, 2005. DOI: 10.1002/joc. 1276 .

HUETE A.; DIDAN K.; MIURA T.; RODRIGUEZ E.P.; GAO X.; FERREIRA L.G. Overview of the radiometric and biophysical performance of the MODIS vegetation índices. Remote Sensing of Environment, v.83, p.195-213, 2002. DOI: 10.1016/ S0034-4257(02)00096-2. 
INSTITUTO NACIONAL DE PESQUISAS ESPACIAIS. Ambdata: variáveis ambientais para modelagem de distribuição de espécies. 2013. Disponível em: <http://www.dpi.inpe.br/ ambdata>. Acesso em: 17 abr. 2015.

LORENZI, H. Árvores brasileiras: manual de identificação e cultivo de plantas arbóreas nativas do Brasil. 2.ed. Nova Odessa: Instituto Plantarum, 1998. v.1, 352p.

LORENZI, H. Árvores brasileiras: manual de identificação e cultivo de plantas arbóreas nativas do Brasil. 4.ed. Nova Odessa: Instituto Plantarum, 2002. v.1, 368p.

MELLO, J.M. de; SCOLFORO, J.R.; CARVALHO, L.M.T. de (Ed.). Inventário florestal de Minas Gerais: Floresta Estacional Decidual: florística, estrutura, diversidade, similaridade, distribuição diamétrica e de altura, volumetria, tendências de crescimento e manejo florestal. Lavras: UFLA, 2008. 265p.

NATIONAL AERONAUTICS AND SPACE ADMINISTRATION. MODIS: Moderate Resolution Imaging Spectroradiometer. Available at: <http://modis.gsfc.nasa.gov/>. Accessed on: Jan. 20 2014.

OLIVEIRA FILHO, A.T. Estudos ecológicos da vegetação como subsídios para programas de revegetação com espécies nativas: uma proposta metodológica. Cerne, v.1, p.64-72, 1994.

PEARSON, R.G.; RAXWORTHY, C.J.; NAKAMURA, M.; PETERSON, A.T. Predicting species distributions from small numbers of occurrence records: a test case using cryptic geckos in Madagascar. Journal of Biogeography, v.34, p.102-117, 2007. DOI: 10.1111/j.1365-2699.2006.01594.X.

PETERSON, A.T.; NAKAZAWA, Y. Environmental data sets matter in ecological niche modelling: an example with Solenopsis invicta and Solenopsis richteri. Global Ecology and Biogeography, v.17, p.135-144, 2008. DOI: 10.1111/j.1466-8238 .2007.00347.x.

PHILLIPS, S.J.; ANDERSON, R.P.; SCHAPIRE, R.E. Maximum entropy modeling of species geographic distributions. Ecological Modelling, v.190, p.231-259, 2006. DOI: 10.1016/j. ecolmodel.2005.03.026.

PHILLIPS, S.J.; DUDÍK, M. Modeling of species distributions with Maxent: new extensions and a comprehensive evaluation. Ecography, v.31, p.161-175, 2008. DOI: 10.1111/j.0906-7590.20 08.5203.x.

PILON, N.A.L.; UDULUTSCH, R.G.; DURIGAN, G. Padrões fenológicos de 111 espécies de Cerrado em condições de cultivo. Hoehnea,v.42,p.425-443,2015.DOI:10.1590/2236-8906-07/2015.
SCOLFORO, J.R.; MELLO, J.M. de; OLIVEIRA, A.D. de (Ed.). Inventário Florestal de Minas Gerais: Cerrado: florística, estrutura, diversidade, similaridade, distribuição diamétrica e de altura, volumetria, tendências de crescimento e áreas aptas para manejo florestal. Lavras: UFLA, 2008a. 816p.

SCOLFORO, J.R.; MELLO, J.M. de; SILVA, C.P. de C. (Ed.). Inventário florestal de Minas Gerais: Floresta Estacional Semidecidual e Ombrófila: florística, estrutura, diversidade, similaridade, distribuição diamétrica e de altura, volumetria, tendências de crescimento e áreas aptas para o manejo florestal. Lavras: UFLA, 2008b. 1029p.

SILVA, C.M.; SILVA, C.I. da; HRNCIR, M.; QUEIROZ, R.T. de; IMPERATRIZ-FONSECA, V.L. Guia de plantas visitadas por abelhas na Caatinga. Fortaleza: Fundação Brasil Cidadão, 2012. 98 .

SOUZA, L.M. de; FARIA, R.A.V.B.; BOTELHO, S.A.; FONTES, M.A.L.; FARIA, J.M.R. Potencial de regeneração natural como método de restauração do entorno de nascente perturbada. Cerne, v.18, p.565-576, 2012. DOI: 10.1590/S0104-77602012000400006.

SOUZA, M.C.S. de; PIÑA-RODRIGUES, F.C.M. Desenvolvimento de espécies arbóreas em sistemas agroflorestais para recuperação de áreas degradadas na Floresta Ombrófila Densa, Paraty, RJ. Revista Árvore, v.37, p.89-98, 2013. DOI: 10.1590/ S0100-67622013000100010.

TRABUCCO, A.; ZOMER, R.J. Global high-resolution soil water balance [dataset]. Washington: CGIARCSI, 2010. Available at: <http://www.cgiar-csi.org/data/ global-high-resolution-soil-water-balance>. Accessed on: Jan. 14 2014.

VALE, I. do; COSTA, L.G.S.; MIRANDA, I.S. Espécies indicadas para a recomposição da Floresta Ciliar da sub-bacia do Rio Peixe-Boi, Pará. Ciência Florestal, v.24, p.573-582, 2014. DOI: 10.5902/1980509815736.

VALE, V.S. do; SCHIAVINI, I.; LOPES, S. de F.; DIAS NETO, O.C.; OLIVEIRA, A.P. DE; GUSSON, A.E. Composição florística e estrutura do componente arbóreo em um remanescente primário de floresta estacional semidecidual em Araguari, Minas Gerais, Brasil. Hoehnea, v.36, p.417-429, 2009. DOI: 10.1590/ S2236-89062009000300003.

WARREN, D.L.; GLOR, R.E.; TURELLI, M. Environmental niche equivalency versus conservatism: quantitative approaches to niche evolution. Evolution, v.62, p.2868-2883, 2008. DOI: 10.1111/j.15 58-5646.2008.00482.x.

Recebido em 7 de agosto de 2015 e aprovado em 19 de janeiro de 2016 